\title{
Cigarette Smoking Induced Inflammation accompany the earliest Acute Brain Injury is the pathological basis of COPD comorbid MDD
}

\author{
Ruining $\mathrm{Hu}^{1}$, Lingzhu Chen ${ }^{1}$, Liang Yuan ${ }^{1}$, Lan Wang ${ }^{1}$, Wenju $\mathrm{Lu}^{1}$, and Qinghui Huang ${ }^{1}$ \\ ${ }^{1}$ Guangzhou Medical University
}

May 12, 2020

\begin{abstract}
Background and Purpose: COPD is one of the most common respiratory diseases worldwide with high comorbidities as major depressive disorder(MDD). Cigarette smoking(CS) is the most major risk factor and smokers tend to be more easy to get COPD and MDD, the mechanisms still remained poorly defined. Experimental Approach: The gene expression microarray analyzed between COPD lung tissues and MDD brain tissues were conducted and enriching GO and KEGG pathways analysis in R. CS exposure induced COPD animal model in mice was established and followed the expression of hub-bottleneck genes. Key Results: Based on microarray analysis, found some DEGs plays a prominent role in COPD and MDD, such as Casitas B-lineage Lymphoma Proto-OncogeneB (CBLB) is involved in the regulation of immune response. Myocyte enhancer factor 2C (MEF2C) have been associated with severe cognitive disability, stereotypic movements and cerebral malformation. Mitogenactivated protein kinase 7 (MAPK7) was involved in various cellular processes. Furthermore, animal studies showed that mice exposure to CS whose airway inflammation increased and inflammatory cells rise in bronchoalveolar lavage fluid accompanied with markedly declined lung function. In addition, CBLB and MAPK7 with abnormal expression in the brain tissue. Even more important, mice exposure to CS whose brain was seriously injured and the nerve cells morphological have changed, CBLB deficiency increased the GM-CSF signal pathway related inflammatory reactions in brain tissue. Conclusion and Implications: In conclusion, CS causes COPD accompanied with early acute brain injury, GM-CSF and MAPK7 signal pathway may be involved in it. To some extent, the study revealing that inflammatory response accompany with the earliest acute brain injury is the pathological basis of COPD complicating MDD
\end{abstract}

\section{Hosted file}

Cigarette Smoking Induced Inflammation accompany the \selectlanguage\{ngerman\} earliest Acute Brain Injur available at https://authorea.com/users/320913/articles/450321-cigarette-smoking-inducedinflammation-accompany-the-earliest-acute-brain-injury-is-the-pathological-basis-ofcopd-comorbid-mdd

\section{Hosted file}

Figures and legends.doc available at https://authorea.com/users/320913/articles/450321cigarette-smoking-induced-inflammation-accompany-the-earliest-acute-brain-injury-is-thepathological-basis-of-copd-comorbid-mdd

\section{Hosted file}

Cigarette Smoking Induced Inflammation accompany the earliest Acute Brain Injury is the pathological ba available at https://authorea.com/users/320913/articles/450321-cigarette-smoking-inducedinflammation-accompany-the-earliest-acute-brain-injury-is-the-pathological-basis-ofcopd-comorbid-mdd 